\title{
The Sommerfeld ground-wave limit for a molecule adsorbed at a surface
}

Li Chen ${ }^{2 *} \dagger$, Jascha A. Lau ${ }^{1,2}+$, Dirk Schwarzer ${ }^{2}$, Jörg Meyer ${ }^{3}$, Varun B. Verma ${ }^{4}$, Alec M. Wodtke 1,2,5 $^{1,5}$

${ }^{1}$ Institute for Physical Chemistry, University of Göttingen, Tammannstr. 6, 37077 Göttingen, Germany

${ }^{2}$ Department of Dynamics at Surfaces, Max-Planck Institute for Biophysical Chemistry, Am Faßberg 11, 37077 Göttingen, Germany

${ }^{3}$ Leiden Institute of Chemistry, Gorlaeus Laboratories, Leiden University, P.O. Box 9502, 2300

RA Leiden, The Netherlands

${ }^{4}$ National Institute of Standards and Technology, Boulder, Colorado, USA

${ }^{5}$ International Center for Advanced Studies of Energy Conversion, Georg-August University of Göttingen, Tammannstraße 6, 37077 Göttingen, Germany

$\dagger$ Present address: State Key Laboratory of Molecular Reaction Dynamics, Dalian Institute of Chemical Physics, Chinese Academy of Sciences, Dalian, Liaoning 116023, People's Republic of China

†These authors contributed equally to this work

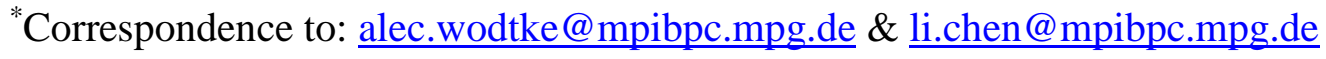

\begin{abstract}
$\underline{\text { Abstract }}$
Using a mid-infrared emission spectrometer based on a superconducting nanowire single-photon detector (SNSPD), we observe the dynamics of vibrational energy pooling of CO adsorbed at the surface of a $\mathrm{NaCl}$ crystal. After exciting a majority of the $\mathrm{CO}$ molecules to their first vibrationally excited state $(\mathrm{v}=1)$, we observe infrared emission from states up to $\mathrm{v}=27$. Kinetic Monte Carlo simulations show that vibrational energy collects in a few $\mathrm{CO}$ molecules at the expense of those up to eight lattice sites away by selective excitation of $\mathrm{NaCl}^{\prime}$ s transverse phonons. The vibrating $\mathrm{CO}$ molecules behave like classical oscillating dipoles, losing their energy to $\mathrm{NaCl}$ lattice-vibrations via the electromagnetic near-field. This is analogous to Sommerfeld's description of the Earth's influence on radio transmission by ground waves.
\end{abstract}

\section{Main Text}

Polar molecules in optical lattices formed by interfering laser beams are platforms for studying quantum magnetism (1), quantum many-body dynamics (2) and quantum computing (3, 4). The electric fields at a crystalline surface are another form of lattice, one capable of orienting and ordering polar molecules. Hence, adsorbing molecules to low-temperature solids might be a complementary and, so far, unexplored approach to studying the lattice dynamics of polar molecules. Unfortunately, dynamical interactions between adsorbates and solid substrates are 
typically much stronger than those between adsorbed molecules $(5,6)$. For example, an adsorbate's vibrational energy may flow to a solid's electrons within picoseconds $(7,8)$ or, due to intrinsically anharmonic interatomic forces, to lattice vibrations within nanoseconds $(9,10)$.

One exception is a monolayer of $\mathrm{CO}$ adsorbed to $\mathrm{NaCl}(100)$ - see Fig. 1c. Here, dipole-dipole interactions between $\mathrm{CO}$ molecules are stronger than $\mathrm{CO}-\mathrm{NaCl}$ interactions, conditions that lead to vibrational energy pooling (VEP). Chang et al. observed VEP producing CO in states at least up to $\mathrm{v}=15$ by near-resonant vibration-to-vibration $(\mathrm{V}-\mathrm{V})$ energy transfer.

$$
\begin{array}{cc}
\mathrm{CO}(1)+\mathrm{CO}(1) \rightarrow \mathrm{CO}(2)+\mathrm{CO}(0) & +\Delta \varepsilon(2) \\
\mathrm{CO}(2)+\mathrm{CO}(1) \rightarrow \mathrm{CO}(3)+\mathrm{CO}(0) & +\Delta \varepsilon(3) \\
\vdots & \vdots \\
\mathrm{CO}(\mathrm{v})+\mathrm{CO}(1) \rightarrow \mathrm{CO}(\mathrm{v}+1)+\mathrm{CO}(0) & +\Delta \varepsilon(\mathrm{v}+1)
\end{array}
$$

Unfortunately, detailed studies were impossible due to the low sensitivity and poor time-response of infrared detectors available at that time (11).

In this work, we detect time- and wavelength-resolved laser-induced infrared fluorescence with a superconducting nanowire single-photon detector (SNSPD) $(12,13)$ in order to study VEP in detail. Kinetic Monte Carlo (kMC) simulations (14-18) of our observations reveal V-V energy transfer occurring between $\mathrm{CO}$ molecules separated by more than eight lattice sites and show that the excess energy represented by $\Delta \varepsilon(\mathrm{v}+1)$ in Eq. (1) is selectively absorbed by NaCl's transverse phonons. Surprisingly, the vibrating CO molecules behave like classical oscillating dipoles, losing their energy to $\mathrm{NaCl}$ lattice-vibrations via the electromagnetic near-field. These rates are quantitatively described by a theory $(19,20)$ that has its origins in Sommerfeld's 1909 description of a radio transmitter interacting with the Earth forming damped electromagnetic surface waves (21). This is a weak coupling limit where the anharmonic interatomic forces normally so important to energy flow can be completely neglected.

Figures 1A \& B show infrared spectra of $\mathrm{CO}$ adsorbed to $\mathrm{NaCl}$ obtained in absorption (panel A) and with laser-induced infrared fluorescence (panel B). The spectrum of the $\mathrm{CO}$ monolayer is composed of a doublet centered at $2052 \mathrm{~cm}^{-1}$, where the intensity pattern is polarization sensitive. The feature at $2055 \mathrm{~cm}^{-1}$ results from the symmetric stretching of the two coupled CO molecules in the $2 \times 1$ unit cell (shown as a dashed rectangle in panel C) $(22,23)$. The $2049 \mathrm{~cm}^{-1}$ line observed with s- and p-polarization arises from the anti-symmetric stretch vibration. For comparison, panel $\mathrm{B}$ shows the laser-induced infrared fluorescence excitation spectrum obtained from a $\mathrm{CO}$ monolayer for both $\mathrm{p}$ - and s- polarization. There can be no doubt that the laser-induced fluorescence results from the excitation of $\mathrm{CO}$ molecules in the monolayer.

Figure $2 \mathrm{~A} \& \mathrm{~B}$ show the experimentally obtained fluorescence emission spectrum (in black) compared to kMC simulations (in red). All features in these spectra result from the first overtone emission of vibrationally excited $\mathrm{CO}$; the emitting vibrational state is indicated by combs. Intensity peaks reflecting enhanced vibrational populations are seen near $v=7,16$ and 25 ; hereafter, we refer to vibrational states near these three values of $\mathrm{v}$ as base-camp 1,2, and 3, respectively.

The red curve in Fig. 2A shows a kMC simulation under our experimental conditions where only nearest neighbor V-V energy transfer is permitted, an assumption used in previous work (14-18). 
This approach yields a peak in population at $v \sim 8$ (base-camp 1), strongly resembling Fig. 3 of Ref.'s (14) and (15) but markedly different than experiment. Note that a single molecule with only 4 nearest neighbors can still reach $\mathrm{v}=8$ since the nearest neighbors can transport vibrational quanta from more distant molecules by process (2).

$$
\mathrm{CO}(1)+\mathrm{CO}(0) \rightarrow \mathrm{CO}(0)+\mathrm{CO}(1) \quad \Delta \varepsilon(1)=0
$$

Population in states higher than $\mathrm{v} \sim 8$ is prevented by a one-phonon energy cut-off $(14,15)$ that is reached when $\Delta \varepsilon(v+1)$ - see Eq. (1) - exceeds the energy of the highest frequency phonon of the $\mathrm{NaCl}$ substrate. Clearly, the nearest neighbor assumption in these kMC simulations fails to describe the experiment.

To produce molecules in higher vibrational states, long distance interactions between vibrationally excited molecules are needed. When vibrational energy pooling is modelled including V-V exchange over an area of $\sim 1000 \AA^{2}$, kMC simulations reproduce experiment well (Fig. 2B, red curve). In this case, vibrationally excited molecules in base-camp 1 states can interact with one another even though they are not likely to be nearest neighbors. For example, processes like

$$
\begin{aligned}
& \mathrm{CO}(7)+\mathrm{CO}(7) \rightarrow \mathrm{CO}(8)+\mathrm{CO}(6) \\
& \mathrm{CO}(8)+\mathrm{CO}(7) \rightarrow \mathrm{CO}(9)+\mathrm{CO}(6) \\
& \vdots \\
& \mathrm{CO}(\mathrm{v})+\mathrm{CO}(7) \rightarrow \mathrm{CO}(\mathrm{v}+1)+\mathrm{CO}(6)
\end{aligned}
$$

allow molecules in base-camp 1 states to climb to base-camp 2, where again the one-phonon energy cut-off slows further pooling. Subsequently, molecules in base-camp 2 climb to base-camp 3 by even longer range interactions. Vibrational states higher than $\mathrm{v}=27$ are not seen as energy transfer to the lowest lying excited electronic states becomes possible (V-E energy transfer). The temporal sequence of base-camp formation can also be seen by taking snap shots of the vibrational distribution in the kMC simulations at different times (see Fig. 2C). This shows that base-camp 1 forms within $100 \mathrm{~ns}$, base-camp 2 within $0.1-1 \mu$ s and base-camp 3 only after 10-100 $\mu \mathrm{s}$.

The distance dependence of dipole-dipole interactions explains the sequential formation of basecamps. From the kMC simulations of Fig. 2B, we find that the average distance between pooling molecules forming base-camp 1 is $4.1 \AA$ (approx. 1 lattice constant); whereas, for base-camp 2 this distance is $11.7 \AA$ and for base-camp 3 it is $17.6 \AA$. One-phonon-assisted V-V rates scale with distance as $R^{-8}$ - see supplementary material - meaning base-camp 1 is formed $10^{3}$ times faster than base-camp 2 which is formed $10^{2}$ times faster than base-camp 3 . This hierarchy of rates is consistent with our experimental observations.

VEP selectively excites transverse $\mathrm{NaCl}$ phonons. Figure 3 (left panel) shows four $\mathrm{kMC}$ simulations (red, blue, green \& brown) of emission spectra using different assumptions about the solid's phonon density of states and compares them to experiment (black). (The assumed phonon density of states used in each simulation appears in the right panel.) While all four simulations resemble experiment, we find best agreement with experiment when only transverse phonons are allowed to accept energy in the ladder climbing process. In fact, only here do we see the formation of three base-camps. 
Under conditions of this work, VEP rapidly produces $\mathrm{CO}$ in many vibrationally excited states. Relaxation of the system back to vibrational equilibrium proceeds more slowly - see Fig. 4A where we show measurements of the time-resolved infrared fluorescence (open symbols) from seven vibrational states. Asymptotically, they all exhibit exponential decay (solid lines) with effective lifetimes, shown as open circles with error bars in Fig. 4B. For kMC simulations of the asymptotic exponential fall-off, we include three elementary processes: $\mathrm{V}-\mathrm{V}$ energy transfer between $\mathrm{CO}$ molecules,

$$
\mathrm{CO}\left(\mathrm{v}^{\prime}\right)+\mathrm{CO}\left(\mathrm{v}^{\prime \prime}\right) \rightarrow \mathrm{CO}\left(\mathrm{v}^{\prime}+1\right)+\mathrm{CO}\left(\mathrm{v}^{\prime \prime}-1\right)
$$

spontaneous radiative emission,

$$
\mathrm{CO}\left(\mathrm{v}^{\prime}\right) \rightarrow \mathrm{CO}\left(\mathrm{v}^{\prime}-1\right)+\mathrm{h} v_{\mathrm{IR}}
$$

and vibrational energy transfer to the $\mathrm{NaCl}$ lattice vibrations.

$$
\mathrm{CO}\left(\mathrm{v}^{\prime}\right) \rightarrow \mathrm{CO}\left(\mathrm{v}^{\prime}-1\right)+\Delta \mathrm{E}_{\mathrm{phonon}}
$$

For process (4) we use the same approach that allowed successful simulation of the data of Fig.'s 2 and 3. See Section F of the SI. For process (5), we use the known radiative emission rate constants for gas-phase $\mathrm{CO}\left(\mathrm{v}^{\prime}\right)$. For process (6) we have tested two models of vibrational energy transfer. The solid squares in Fig. 4B are the effective lifetimes that result from implementation of the Skinner-Tully (ST) model described in detail in section F of the SI (14) - here, anharmonic coupling of $\mathrm{CO}$ vibration to $\mathrm{NaCl}$ phonons is mediated via the $\mathrm{CO}-\mathrm{NaCl}$ surface bond (14-18). The predicted effective lifetimes are in poor agreement with experiment and they exhibit a vibrational quantum number dependence that is far too strong. (Note the logarithmic scale).

We also tested a model developed by Chance, Prock and Silbey (CPS) (19, 20), shown as filled circles in Fig. 4B. The CPS theory is briefly described in section F4 of the SI. The agreement with experiment is striking. CPS was developed to describe fluorescence lifetimes of dye molecules interacting through an inert spacer layer with an absorbing and reflecting solid (24-29). Here, coupling occurs through electromagnetic fields. The fact that CPS accurately reproduces the observations of this work, suggests that $\mathrm{CO}$ vibrational relaxation to $\mathrm{NaCl}$ lattice-vibration also occurs through the electromagnetic near-field despite the fact that there is a surface bond.

We emphasize that the weak vibrational quantum number dependence of the effective lifetime is indicative of coupling via the electromagnetic field - ST predicts a change in effective lifetime of four orders of magnitude over the same range of $\mathrm{v}$ where CPS predicts less than a ten-fold change. Of course, the ST model could in future be improved, an ab initio treatment of the coupling to the solid's phonon bath is still lacking. Despite this, we expect the strong v-dependence to be retained - see SI. Referring to Fig. 4B, we speculate that the steeper slope above v 23 indicates a transition to ST behavior.

Normally, we consider energy flow within an ensemble of oscillators to be a consequence of interatomic anharmonicity. This work shows that we can bind a molecule to a solid with sufficient strength to create samples that are stable over long periods of time without any influence of anharmonicity on the vibrational energy relaxation. In this Sommerfeld ground-wave limit, vibrational relaxation occurs exclusively via the electromagnetic field obeying the CPS model. 


\section{4}

155

156

157
Here, the strength of coupling scales with the solid's imaginary index of refraction and the square of the molecule's transition dipole moment - see SI. Besides $\mathrm{CO}$ on $\mathrm{NaCl}$, other similar systems are to be expected. $\mathrm{CO}$ on $\mathrm{KCl}$ and $\mathrm{N}_{2}$ on $\mathrm{NaCl}$ are both interesting possibilities, whose CPS coupling would be even weaker than seen here. For dipolar adsorbates that find themselves within this limit, the solid's crystalline lattice can be exploited to produce spatial registry and orientational order while the strength of dipole-dipole interactions between the adsorbate molecules still far exceeds the adsorbate coupling to the solid. The prospect to study quantum lattice dynamics in systems like this appears promising.

Supplementary Content includes: 1) Materials and Methods, 2) Supplementary Text, 3) Figs. S1 to S6 and 4) References (32-48)

\section{References and Notes}

[1] J. Park et al., Science 357, 372 (2017).

[2] B. Yan et al., Nature 501, 521 (2013).

[3] D. DeMille, Phys Rev Lett 88, 4 (2002).

[4] A. Andre et al., Nat Phys 2, 636 (2006).

[5] K. Golibrzuch et al., Ann Rev Phys Chem 66, 399 (2015).

[6] A. M. Wodtke, Chem Soc Rev 45, 3641 (2016).

[7] J. D. Beckerle et al., Phys Rev Lett 64, 2090 (1990).

[8] P. R. Shirhatti et al., Nat Chem 10, 592 (2018).

[9] P. Guyotsionnest et al., Phys Rev Lett 64, 2156 (1990).

[10] K. Lass et al., J Chem Phys 123, 4 (2005).

[11] H. C. Chang et al., Phys Rev Lett 65, 2125 (1990).

[12] L. Chen et al., Acc Chem Res 50, 1400 (2017).

[13] L. Chen et al., Optics Express 26, 14859 (2018).

[14] S. A. Corcelli et al., J Chem Phys 116, 8079 (2002).

[15] S. A. Corcelli et al., J Phys Chem A 106, 10849 (2002).

[16] E. T. D. Boney et al., J Chem Phys 139, (2013).

[17] S. A. Corcelli, Yale University, (2002).

[18] S. A. Egorov et al., J Chem Phys 103, 1533 (1995). 
173 [19] R. R. Chance et al., Adv Chem Phys 37, 1 (1978).

174 [20] R. R. Chance et al., J Chem Phys 60, 2744 (1974).

175 [21] A. Sommerfeld, Ann. Phys.-Leip. 28, 665 (1909).

176 [22] J. Heidberg et al., J Chem Phys 95, 9408 (1991).

177 [23] D. Schmicker et al., J Chem Phys 95, 9412 (1991).

178 [24] K. H. Drexhage et al., Ber Bunsenges Phys Chem 70, 1179 (1966).

179 [25] K. H. Drexhage, Scientific American 222, 108 (1970).

180 [26] K. H. Drexhage et al., Ber Bunsenges Phys Chem 72, 329 (1968).

181 [27] H. Kuhn, Naturwissenschaften 54, 429 (1967).

182 [28] H. Kuhn, J Chem Phys 53, 101 (1970).

183 [29] K. H. Tews et al., Nature 228, 276 (1970).

184 [30] H. C. Chang et al., J Chem Phys 89, 7561 (1988).

185 [31] J. Heidberg et al., J Electron Spectrosc Relat Phenom 64-5, 227 (1993).

186 [32] J. Estel et al., Surf Sci 54, 393 (1976).

187 [33] H. J. Chen et al., Chinese Physics B 24, (2015).

188 [34] J. P. Perdew et al., Phys Rev Lett 77, 3865 (1996).

189 [35] V. Blum et al., Comput. Phys. Commun. 180, 2175 (2009).

190 [36] H. J. Monkhorst et al., Phys. Rev. B 13, 5188 (1976).

191 [37] P. Haas et al., Phys. Rev. B 79, (2009).

192 [38] P. Haas et al., Phys. Rev. B 79, (2009).

193 [39] G. X. Zhang et al., New J Phys 20, (2018).

194 [40] K. Parlinski et al., Phys Rev Lett 78, 4063 (1997).

195 [41] A. Togo et al., Scripta Materialia 108, 1 (2015).

196 [42] R. R. Chance et al., J Chem Phys 62, 2245 (1975).

197 [43] R. R. Chance et al., J Chem Phys 60, 2184 (1974).

198 [44] H. Kuhn et al., Angewandte Chemie-International Edition 10, 620 (1971). 
199 [45] A. Baños, in International series of monographs on electromagnetic waves. (Pergamon Press, $200 \quad$ Oxford, New York, 1966).

201 [46] L. E. Brus, J Chem Phys 74, 737 (1981).

202 [47] A. D. Boese et al., J Phys Chem C 120, 12637 (2016).

203 [48] E. D. P. J. E. Eldridge, Handbook of Optical Constants of Solids. E. D. Palik, Ed., Sodium 204 Chloride (NaCl) (Academic Press, New York, 1997), vol. 1. 


\section{Acknowledgements}

We thank S. A. Corcelli for sharing his kinetic Monte Carlo simulation code and A.

Kandratsenka for helpful discussions. We also thank Prof. Fabian Heidrich-Meisner for providing helpful suggestions after reading an early version of this manuscript. J.M. gratefully acknowledges financial support from The Netherlands Organisation for Scientific Research (NWO) under Vidi Grant No. 723.014.009.

\section{Figures}
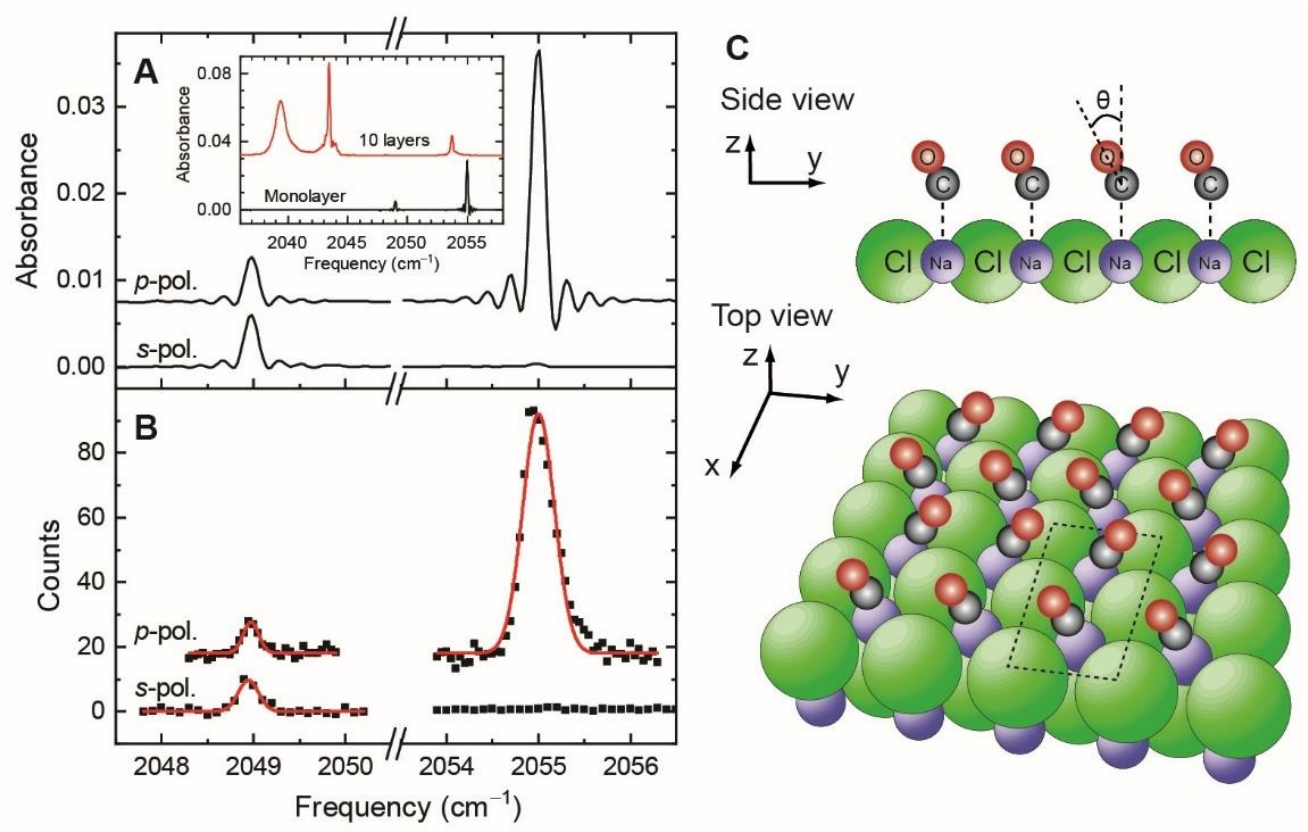

\section{Top view}

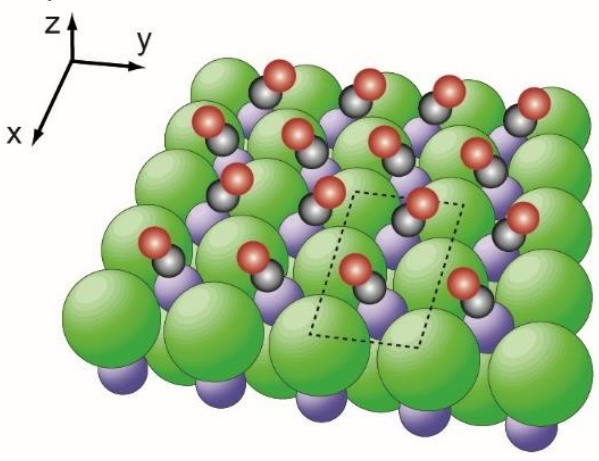

Figure 1 | Structure and infrared spectroscopy of the ${ }^{13} \mathrm{C}^{18} \mathrm{O}$ monolayer on $\mathrm{NaCl}(100)$ : The

polarization dependent monolayer spectrum is observed with (A) FTIR absorbance spectroscopy and (B) laser-induced infrared fluorescence by scanning the laser excitation frequency and integrating the total fluorescence signal between 50 and $1050 \mu$ s after the laser pulse. The spectra were recorded at a surface temperature of $\sim 7 \mathrm{~K}$. Note that the baseline of the p-polarized spectra is shifted for clarity. The inset in panel A shows that the IR absorption spectrum of the $\mathrm{CO}$ monolayer (black) is clearly distinguishable from that of a multilayer (red) for p-polarized light. $\mathrm{CO}$ molecules in the multilayer but not in contact with the $\mathrm{NaCl}$ surface give rise to a doublet centered at $2042 \mathrm{~cm}^{-1}(30,31)$. The feature at $2054 \mathrm{~cm}^{-1}$ arises from the $\mathrm{CO}$ molecules at the buried $\mathrm{NaCl}$ interface (31). The monolayer line intensities have been corrected for an offset of $18^{\circ}$ in the polarization of the FTIR spectrometer light source. In panel B, the experimental data is represented by square symbols and red lines are Gaussian fits to guide the eye. Panel C: Structure of the monolayer $\mathrm{CO}$ on $\mathrm{NaCl}(100)(22,23)$. The nearest neighbour CO-CO distance is $3.96 \AA$. At a surface temperature below $35 \mathrm{~K}$, the $\mathrm{CO}$ molecules are tilted with respect to the surface normal by an angle of $25^{\circ}$, and arranged in antiparallel oriented rows to form a $p(2 \times 1)$ unit cell, as depicted by the dashed rectangle. 


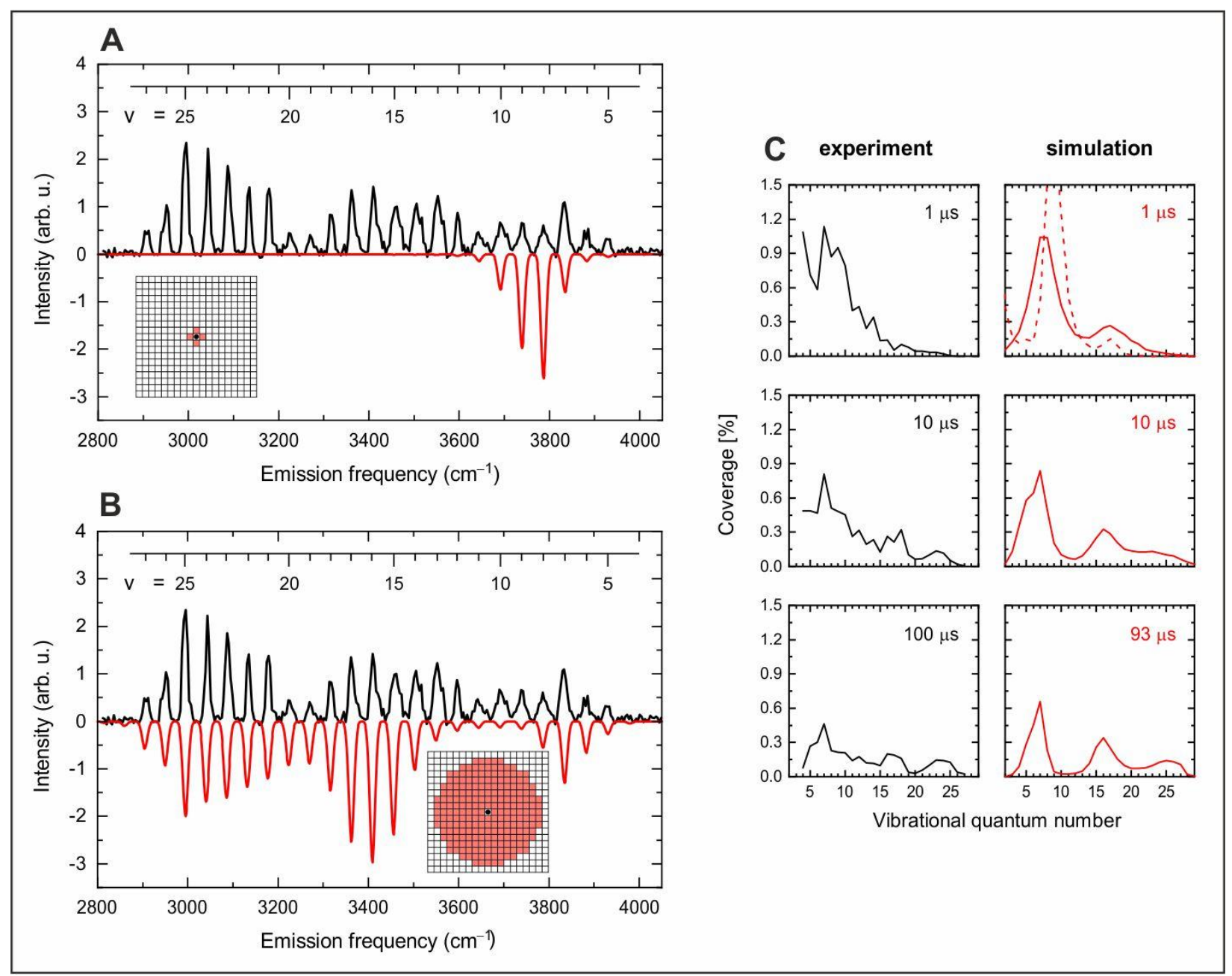

Figure 2 | Base-camp pooling mechanism operating for a monolayer of $\mathrm{CO}$ on $\mathrm{NaCl}(100)$ : In panels

$\mathrm{A}$ and $\mathrm{B}$, we show the experimentally observed emission spectrum (black solid lines) and simulated spectra using kinetic Monte Carlo methods (red solid lines). The insets represent the CO lattice and the red shaded areas visualize the interaction distance around a given $\mathrm{CO}$ molecule (black dot) used in each $\mathrm{kMC}$ simulation. In panel A, the simulation allows only nearest neighbor $\mathrm{V}-\mathrm{V}$ exchange, an assumption that was also made in Ref.'s (14-16). In panel B, the simulation includes molecular interactions out to a distance of $34 \AA$. In each case, the monolayer feature at $2055 \mathrm{~cm}^{-1}$ (Fig. 1B) was excited with a narrowband infrared laser and the emission was dispersed through a monochromator and detected with an SNSPD integrating the signal-counts between 50 and $250 \mu$ s after the laser pulse. Panel C: Snap shots in the experimentally derived and simulated population distributions showing sequential formation of the base-camps. Note that the red dashed line corresponds to the population distribution at $100 \mathrm{~ns}$. 

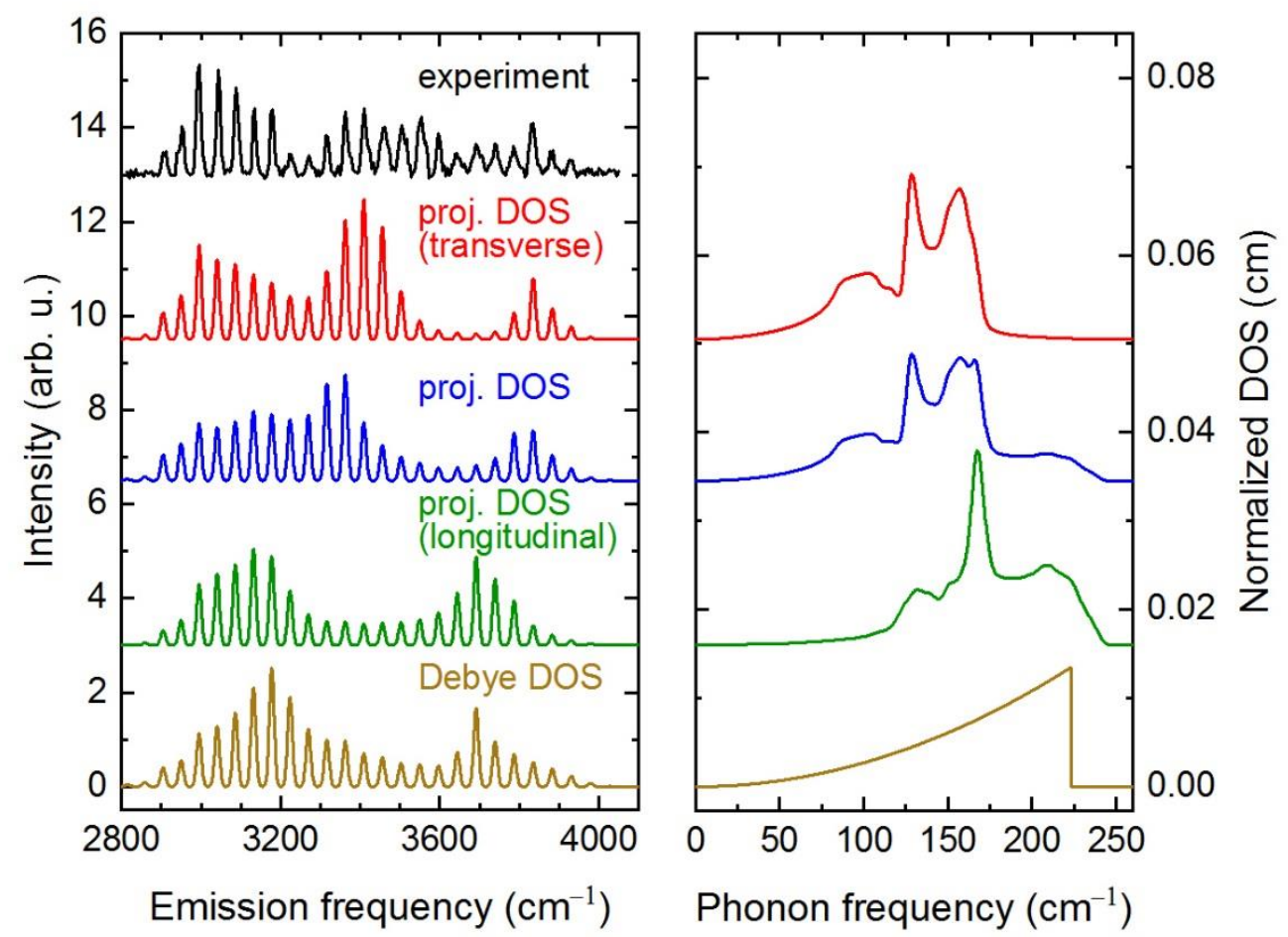

Figure 3 | Selective excitation of $\mathrm{NaCl}$ transverse phonons during energy pooling: The experimental density of states spectra. In each simulation, the $\mathrm{CO}$ - to - $\mathrm{CO}$ interaction distance extends up to $34 \AA$. Each pooling step has an energy release to phonons of the solid; the probability depends on the density of phonon states at that energy. Shown are the results for a Debye DOS (brown) and three DFT-based DOSs: projection of the bulk DOS for $\mathrm{NaCl}$ onto the motion of the $\mathrm{Na}$ ions in the (100) plane (blue), longitudinal contribution to the projected DOS (green), and transverse contribution to the projected DOS (red). 

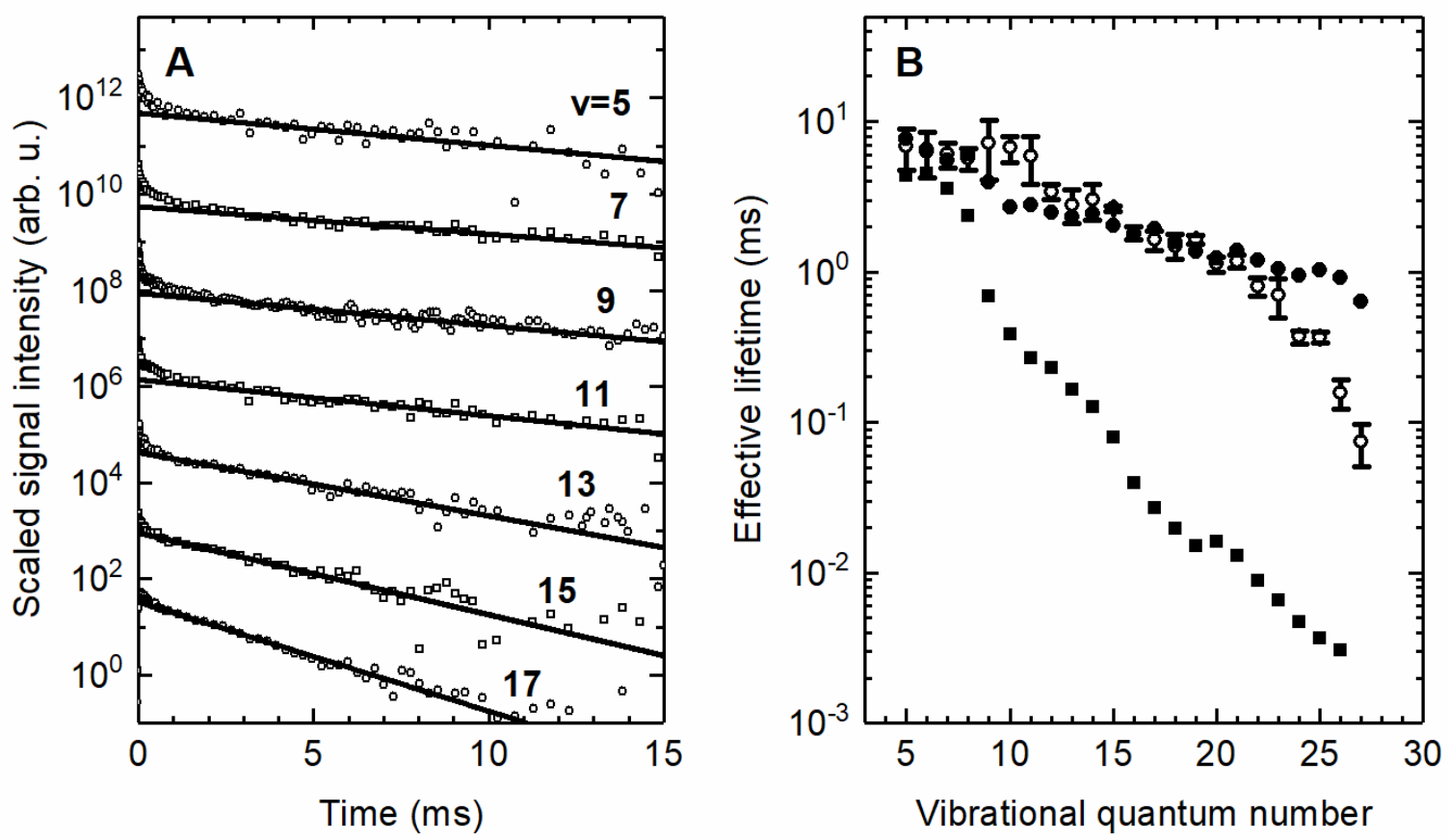

Figure 4 | Relaxation of $\mathrm{CO}$ to the $\mathrm{NaCl}$ solid follows the CPS model: Panel A shows representative temporal profiles of wavelength resolved infrared fluorescence (open symbols); the emitting vibrational state's quantum number is indicated. The long-time relaxation exhibits an exponential decay (black solid lines). Note the $y$-axis is logarithmic and that the data are offset from one another along the y-axis for clarity. The effective exponential lifetime obtained from these (and other) fits are shown in Panel B (black open circles with error bars). The kMC simulations also exhibit long-time exponential behaviour. The corresponding effective lifetimes are shown as solid symbols for two different vibrational relaxation models: the Skinner-Tully model (solid squares) and the CPS model (solid circles). 


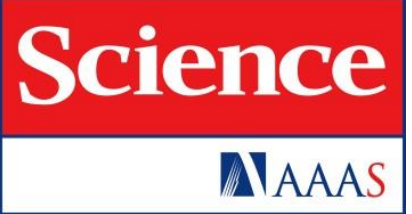

\section{Supplementary Materials for}

The Sommerfeld ground-wave limit for an adsorbed molecule at a surface

Li Chen, Jascha A. Lau, Dirk Schwarzer, Jörg Meyer, Varun B. Verma, Alec M. Wodtke

Correspondence to: alec.wodtke@mpibpc.mpg.de and li.chen@mpibpc.mpg.de

\section{This PDF file includes:}

Materials and Methods

Supplementary Text

Figs. S1 to S6 


\section{Materials and Methods}

Experimental

The experimental apparatus has been described in detail $(12,13)$. Briefly, a monolayer of $\mathrm{CO}$ was prepared on a $\mathrm{NaCl}(100)$ surface by exposing the surface held at $\leq 55 \mathrm{~K}$ to isotopically purified ${ }^{13} \mathrm{C}^{18} \mathrm{O}$ (Sigma-Aldrich, 99\% atom ${ }^{13} \mathrm{C}$ and $99 \%$ atom ${ }^{18} \mathrm{O}$ ) vapor. After the chamber had been evacuated, the sample was cooled to $\sim 7 \mathrm{~K}$ with a closed-cycle liquid Helium refrigerator (RDK-408D2, Sumitomo). A narrow band infrared laser pulse (0.05 cm-1 FWHM bandwidth, 4.7 ns FWHM pulse duration, $10 \mathrm{~Hz}$ repetition rate and $\sim 120 \mu \mathrm{J}$ pulse energy) was focused onto the surface to excite the adsorbed $\mathrm{CO}$ molecules via the fundamental $\mathrm{C}-\mathrm{O}$ stretching vibration at $2055 \mathrm{~cm}^{-1}$. The laser pumping of $\mathrm{CO}(\mathrm{v}=0 \rightarrow \mathrm{v}=1)$ transition is saturated, and a majority of the $\mathrm{CO}(\mathrm{v}=0)$ molecules are vibrationally excited to the $\mathrm{v}=1$ state. Laser-induced infrared fluorescence was dispersed through a home built liquid-nitrogen cooled grating monochromator. The wavelength-resolved temporal profiles were detected using a single photon counting detector based on an amorphous tungsten silicide (a-WSi) superconducting nanowire, whose output was recorded by a multichannel scaler (MCS6A-2, FAST ComTec GmbH).

\section{Kinetic Monte Carlo Simulations}

A rejection-free kinetic Monte Carlo algorithm based on the original FORTRAN code of Ref.'s $(14,17)$ was used. The code has been extended to include vibrational energy pooling beyond next-neighbor distances, overtone fluorescence, V-E transfer and stimulated emission. The simulation results are averaged over 50 trajectories where one trajectory consists of a $100 \times 100$ grid of ${ }^{13} \mathrm{C}^{18} \mathrm{O}$ molecules in a square lattice $(3.96 \AA)$ with periodic boundary conditions. The simulation temperature of $11 \mathrm{~K}$ is chosen slightly above the experimental temperature of 7 $\mathrm{K}$, which is measured next to the $\mathrm{NaCl}$ crystal. Initially all population is assumed to be in $\mathrm{v}=0$ and we let the simulation evolve for about $33 \mathrm{~ms}$ after laser excitation. The highest vibrational state considered is $\mathrm{v}=35$. Rate constants are calculated a priori for the following kinetic elementary steps (see section $\mathrm{F}$ of supplementary text for the corresponding equations): stimulated emission and absorption of the $\mathrm{v}=1 \leftarrow 0$ transition, vibrational energy pooling reactions, radiative and non-radiative vibrational relaxation processes, and V-E transfer for high vibrational states.

The laser excitation rate is simulated by a temporal Gaussian profile with a FWHM of $4.7 \mathrm{~ns}$ and a peak excitation rate of $3.44 \times 10^{9} \mathrm{~s}^{-1}$. The vibrational energy pooling rate constants for the reaction $\mathrm{CO}(\mathrm{n})+\mathrm{CO}(\mathrm{m}) \rightarrow \mathrm{CO}(\mathrm{n}+1)+\mathrm{CO}(\mathrm{m}-1)$ are calculated based on the model presented in Ref.'s $(14,17)$. Since the dipole-dipole interaction potential between the $\mathrm{CO}$ molecules changes only slightly for the tilted $(2 \times 1)$ structure, we assume molecules oriented along the surface normal for convenience. The maximum interaction distance is about $34 \AA$ unless otherwise stated. The integrals over the phononic DOS are calculated numerically considering energy pooling processes involving up to 3 phonons. Since the model assumes that the adsorbed CO molecules follow the surface-parallel motion of the Na ions underneath, a more realistic DOS is used by projecting the bulk DOS for $\mathrm{NaCl}$ onto the motion of the $\mathrm{Na}$ ions in the (100) plane. Radiative relaxation by fluorescence is treated as in Ref.'s $(14,16)$ including fundamental, 1st and 2nd overtone emission. Vibrational relaxation is based on the CPS model for all simulations except for the one shown in Fig. 4 (solid squares). Vibrational relaxation according to the Skinner-Tully model is based on Ref.'s (14-18), without further modifications, i.e., assuming a phonon DOS based on the Debye model coupled with the deformation potential approximation. $\mathrm{V}$-E transfer is included for vibrational states with $\mathrm{v} \geq 28$ using a fixed rate of $1 \times 10^{5} \mathrm{~s}^{-1}$, effectively transferring these vibrational states into $\mathrm{v}=0$. 


\section{Supplementary Text}

\section{A. Preparation of monolayer and multilayer $\mathrm{CO}$ samples on $\mathrm{NaCl}(100)$}

The monolayer and multilayer $\mathrm{CO}$ samples are prepared by exposing the UHV-cleaved $\mathrm{NaCl}(100)$ surface to a backfilling $\mathrm{CO}$ pressure at controlled surface temperatures. Before gas admission, the surface is cleaned by briefly annealing to $390 \mathrm{~K}$ to remove any adsorbates $\left(\mathrm{H}_{2}, \mathrm{H}_{2} \mathrm{O}\right.$, $\mathrm{CO}$ and $\mathrm{CO}_{2}$ ) (32), and the $\mathrm{CO}$ gas sample (Sigma-Aldrich, $99 \%$ atom ${ }^{13} \mathrm{C}$ and $99 \%$ atom ${ }^{18} \mathrm{O}$ ) from a lecture bottle is purified using a liquid nitrogen trap. For preparation of a monolayer sample, the surface temperature is held at $\mathrm{T}_{\mathrm{s}}=55 \mathrm{~K}$ and a leak valve admits $\mathrm{CO}$ into the UHV chamber at a pressure of $1 \times 10^{-6}$ mbar. This is continued until coverage is saturated; i.e., the FTIR absorption band remains unchanged with a further exposure. Next, we turn off the surface heating while slowly closing the leak valve and stop gas admission completely when the surface reaches $\mathrm{T}_{\mathrm{s}}=35 \mathrm{~K}$. We then hold the surface temperature at $35 \mathrm{~K}$ until the chamber pressure is below $5 \times 10^{-10} \mathrm{mbar}$; this prevents growth of $\mathrm{CO}$ overlayers, which is possible only at $\mathrm{T}_{\mathrm{s}}<32 \mathrm{~K}(30,31)$. Subsequently, the surface is cooled to the temperature needed for the experiment; typically $7 \mathrm{~K}$. A multilayer sample is grown epitaxially on top of the monolayer (30) by additional CO dosing at $\mathrm{T}_{\mathrm{s}}<15 \mathrm{~K}$. At a CO background pressure of $1 \times 10^{-6} \mathrm{mbar}$, we observe a growth rate of about 100 -monolayer per minute. After the dosing, the surface is briefly annealed to $25 \mathrm{~K}$ for several minutes to help form an equilibrium crystal structure of the multilayer sample (31).

\section{B. $\mathrm{LiIO}_{3}$ optical parametric amplifier laser}

A tunable dye laser pulse at around $863 \mathrm{~nm}$ (LDS867 in ethanol, 14 mJ, Cobra-Stretch, Sirah Lasertechnik $\mathrm{GmbH})$ and a seeded $1064 \mathrm{~nm}$ laser pulse $(130 \mathrm{~mJ}, 10 \mathrm{~Hz}$, ca. 6 ns pulse width, Continuum Surelite III-10EX) are mixed in a temperature stabilized $\mathrm{LiIO}_{3}$ crystal $\left(100{ }^{\circ} \mathrm{C}\right)$ for difference-frequency generation (DFG). The dye laser is pumped by the $532 \mathrm{~nm}$ second harmonic output of the Nd:YAG laser. The DFG output is tuneable from 1950 to $2650 \mathrm{~cm}^{-1}$ with pulse energies in the range $70 \mu \mathrm{J}$ to $250 \mu \mathrm{J}$.

The IR laser frequency is calibrated with photoacoustic spectroscopy of $\mathrm{CO}$ in a gas cell (ca. 10 mbar). Also from photoacoustic measurements, the bandwidth of the IR laser was determined to be $0.05 \mathrm{~cm}^{-1}$. The pulse duration measured using the SNSPD was $4.7 \mathrm{~ns}$ (FWHM) (13).

\section{Vibrational spectroscopic constants of $\mathrm{CO} / \mathrm{NaCl}(\mathbf{1 0 0})$}

Based on an anharmonic oscillator model of the monolayer $\mathrm{CO}$ vibrational energy levels

$$
E_{\mathrm{v}}=(\mathrm{v}+1 / 2) \omega_{e}-(\mathrm{v}+1 / 2)^{2} \omega_{e} x_{e}+(\mathrm{v}+1 / 2)^{3} \omega_{e} y_{e}
$$

we assign the emission spectrum by fitting the measured overtone emission frequencies (S1) to the following expression:

$$
\tilde{v}_{\mathrm{v} \rightarrow \mathrm{v}-2}=E_{\mathrm{v}}-E_{\mathrm{v}-2}=2 \omega_{e}-(4 \mathrm{v}-2) \omega_{e} x_{e}+6 \mathrm{v}(\mathrm{v}-1) \omega_{e} y_{e}+\frac{7 \omega_{e} y_{e}}{2}
$$

This yields the spectroscopic constants $\omega_{e}=(2074.6 \pm 0.9) \mathrm{cm}^{-1}, \omega_{e} x_{e}=(12.22 \pm 0.04) \mathrm{cm}^{-1}$ and $\omega_{e} y_{e}=(0.012 \pm 0.002) \mathrm{cm}^{-1}$. The uncertainties result from 10 measurements on different days and different $\mathrm{NaCl}(100)$ sample surfaces. We note that the wavelengths identified by Eq. 2 differ from both $\mathrm{CO}$ gas phase and multilayer emission frequencies. 


\section{Vibrational state-resolved temporal profiles of $\mathrm{CO}$ overtone emission}

The fluorescence intensity temporal profiles were measured using the SNSPD (12) together with a multi-channel scaler (MCS6A-2, FAST ComTec GmbH). We determined the time zero by measuring the arrival time of scattered laser light. The monochromator was tuned to each of the detected overtone $(\mathrm{v} \rightarrow \mathrm{v}-2)$ emission lines (main manuscript, Fig. 2). All temporal profiles were recorded in a time range of -1.0 to $50 \mathrm{~ms}$ with a bin-time of $51.2 \mathrm{~ns}$ in order to follow the rapid vibrational energy pooling dynamics as well as the slow relaxation dynamics. The measured raw data were re-binned to achieve satisfactory $\mathrm{S} / \mathrm{N}$ ratio at a cost of reduced time resolution. The acquisition time varied between 6 and 26 minutes depending on the signal intensity.

Fig. S2 presents fluorescence temporal profiles for all the monolayer $\mathrm{CO}$ vibrational states (v=4-27) detected in this experiment on a linear (Fig. S2-A) and logarithmic time-scale (Fig. S2$\mathrm{B})$. The fluorescence rise is faster than $1 \mu \mathrm{s}$ for the lower states $\mathrm{v}=4-12$ and cannot be resolved due to insufficient $\mathrm{S} / \mathrm{N}$.

\section{E. Calibration of absolute vibrational populations}

We convert the measured dispersed fluorescence intensity temporal profiles $I_{n}(t)$ to relative time-dependent populations $P_{n}(t)$ for each vibrational state $n$ as was described in Ref. (12):

$$
P_{n}(\mathrm{t})=\frac{I_{n}(\mathrm{t})}{\eta \cdot k_{\mathrm{f}}}
$$

$\eta$ and $k_{\mathrm{f}}$ are the wavelength dependent system detection efficiency and the fluorescence rate, respectively. Fig. S3 shows the relative error of $P_{n}(\mathrm{t})$ derived from fluorescence spectra integrated over 0.05-1.05 ms.

We estimate the absolute population by comparison with the kinetic Monte Carlo (kMC) simulation. At short times after the laser pulse, the total number of vibrational quanta in the $\mathrm{CO}$ monolayer is conserved because vibrational energy loss is unimportant. In other words, $\sum_{n} n \cdot P_{n}(t) \approx$ const. for $t<10 \mu \mathrm{s}$. From our $\mathrm{kMC}$ simulation, $\sum_{n} n \cdot P_{n}(t=1 \mu \mathrm{s})=0.73$ was determined and used to calculate the absolute time-dependent vibrational populations in Fig. S2.

\section{F. Rate constants for kinetic Monte Carlo (kMC) simulations}

Vibrational dynamics in the $\mathrm{CO}$ monolayer on $\mathrm{NaCl}$ is governed by the interplay of vibrational energy pooling (VEP), fluorescence, non-radiative $\mathrm{CO}$ vibrational energy transfer to the $\mathrm{NaCl}$ substrate and vibration-to-electronic (V-E) energy transfer. We calculate the corresponding rate constants based on available theoretical models. The results are summarized in Fig. S4.

\section{Fluorescence rate constants}

The fluorescence rate constants, $k_{\mathrm{f}}$, are calculated based on standard spectroscopic relationships $(12,14,16)$ using the following parameter values for the monolayer ${ }^{13} \mathrm{C}^{18} \mathrm{O}$ on $\mathrm{NaCl}$ (100): slope of the electric dipole moment function $\mu^{\prime}=3.2 \mathrm{D} / \AA$ (assuming the optical and physical properties of the physisorbed $\mathrm{CO}$ on $\mathrm{NaCl}$ do not change appreciably from those of the gas-phase) 
(33), Morse parameter $a=2.34 \AA^{-1}$, and the spectroscopic constants $\omega_{e}=2074.6 \mathrm{~cm}^{-1}$ and $\omega_{e} x_{e}=$ $12.22 \mathrm{~cm}^{-1}$ (see Section $\left.C\right)$. Note that the calculated rates for $1^{\text {st }}$ overtone $(\Delta v=2)$ and $2^{\text {nd }}$ overtone emission $(\Delta v=3)$ are also included in the kMC simulations but not shown in Fig. S4.

\section{Vibrational energy pooling rate constants}

The VEP rate constants are calculated based on the model from Corcelli and Tully $(14,15,17)$ with a modified treatment of the $\mathrm{NaCl}$ phonon spectrum. Note that there is a minor typographical error in the formula for $k_{n m}^{(p)}$ in Ref. (14) but Corcelli's PhD thesis (17) shows the correct equation. The pooling rate constant, $k_{n m}$, for a pooling reaction $\mathrm{CO}(\mathrm{n})+\mathrm{CO}(\mathrm{m}) \rightarrow \mathrm{CO}(\mathrm{n}+1)+\mathrm{CO}(\mathrm{m}-1)$ for a given distance $R$ (in SI units) is:

$$
\begin{gathered}
k_{n m}=\sum_{p=1}^{p_{\max }} k_{n m}^{(p)} \\
k_{n m}^{(p)}=\frac{2 \pi}{\hbar} p ! f_{p}^{2} 2^{p}|\langle n|x| n+1\rangle|^{2}|\langle m|x| m-1\rangle|^{2}\left(\frac{\left(n\left(E_{n m} / p\right)+1\right) \hbar}{2 M}\right)^{p} I_{p} \\
f_{p}=(-1)^{p} \frac{\mu^{\prime 2}(p+1)(p+2)}{\left(4 \pi \varepsilon_{0}\right) 2 R^{p+3}} \\
I_{p}=\int_{0}^{\infty} \mathrm{d} E_{1} \cdots \int_{0}^{\infty} \mathrm{d} E_{p} \frac{\rho\left(E_{1}\right)}{E_{1}} \cdots \frac{\rho\left(E_{p}\right)}{E_{p}} \delta\left(E_{n m}-E_{1}-\cdots-E_{p}\right) \\
n(E)=\left(\exp \left(\frac{E}{k_{B} T}\right)-1\right)^{-1}
\end{gathered}
$$

where $p$ is the number of phonons involved and $p_{\max }=3$, since processes involving more than 3 phonons do not yield significant rate constants for all $\mathrm{n}-\mathrm{m}$ transitions that are of relevance here (14). $M$ is the mass of a $\mathrm{Na}$ atom ( $23 \mathrm{amu}$ ). The matrix elements of the relative bond coordinate $x$ are calculated as for the fluorescence rate constants (see Ref.'s $(12,14,16,33)$ ). The electric dipole moment function is assumed to be linear with a slope of $\mu^{\prime}=3.2 \mathrm{D} / \AA$, for the same reason as in the calculation for the fluorescence rate constants. The integrals $I_{p}$ are evaluated numerically for a given phonon density of states (DOS), $\rho(E)$, which is normalized such that $\int_{0}^{\infty} \mathrm{d} E \rho(E)=1$, where the energy mismatch $E_{n m}$ is positive for exothermic reactions. Rate constants for reverse endothermic reactions are derived from the rate constants of the exothermic reactions by detailed balance.

Phonons for bulk $\mathrm{NaCl}$ in the rock-salt structure are calculated based on density functional theory (DFT) using the exchange-correlation functional due to Perdew, Burke and Ernzerhof (34) as implemented in the FHI-aims all electron DFT package (35). Tight settings are used for the basis sets and integration grids together with equivalents of a $4 \times 4 \times 4$ Monkhorst-Pack grid (36) for the Brillouin zone sampling in the primitive unit cell. These settings yield a perfectly converged lattice constant of $5.698 \AA$ in excellent agreement with earlier all-electron DFT calculations (37-39) and a well-converged phonon DOS. 
Force constants are calculated according to the finite displacement method (40) in $4 \times 4 \times 4$ supercells of the primitive unit cell with optimal exploitation of symmetry as implemented in the phonopy code (41). The total phonon DOS is then obtained based on a Fourier-interpolated $40 \times 40 \times 40$ grid of phonon-wave vectors $\mathbf{q}$ for all six phonon bands $i$, where $E_{\mathbf{q} i}$ is the corresponding energy equivalent of each phonon:

$$
\rho^{\text {total }}(E)=\frac{1}{6} \sum_{\mathbf{q}} \sum_{i=1}^{6} \delta\left(E-E_{\mathbf{q} i}\right)
$$

Here and in the following, the delta functions are broadened by Gaussians with a very small width of only $2.42 \mathrm{~cm}^{-1}$ thanks to very dense q-grid. Fig. S5 shows the results of the phonon DOS calculations used in this work.

The model by Corcelli and Tully is based on the modulation of the dipole-dipole interaction between two $\mathrm{CO}$ molecules. This is caused by phonons in the $\mathrm{NaCl}$ surface, that change the lateral distance between two COs. These are phonons that involve lateral movement of the $\mathrm{Na}$ atom directly beneath each $\mathrm{CO}$ molecule (see Fig. S6). Consequently, we use a phonon DOS that is projected onto phonons which include Na-atom displacements in the (100) plane. Furthermore, using the scalar product between the atom-wise displacement and the wave vectors of each phonon, we construct the projected phonon DOS for transverse $(\mathrm{T})$ or longitudinal $(\mathrm{L})$ phonons

$$
\rho^{\mathrm{Na},(100) ; \mathrm{T}, \mathrm{L}}(E)=\frac{1}{3} \sum_{\mathbf{q}} \sum_{i=1}^{6} \delta\left(E-E_{\mathbf{q} i}\right)\left|\mathbf{p}_{\mathbf{q} i}^{\mathrm{Na} ; \mathrm{T}, \mathrm{L}}\right|^{2} .
$$

$\mathbf{p}_{\mathbf{q} i}^{\mathrm{Na} ; \mathrm{T}, \mathrm{L}}$ is the transverse or longitudinal contribution of the normalized displacement vector $\mathbf{e}_{\mathbf{q} i}=\left(\mathbf{e}_{\mathbf{q} i}^{\mathrm{Na}}, \mathbf{e}_{\mathbf{q} i}^{\mathbf{C l}}\right)$ for $\mathrm{Na}$ atoms only, i.e., entries for $\mathbf{e}_{\mathbf{q} i}^{\mathrm{Cl}}$ are set to zero:

$$
\begin{gathered}
\mathbf{p}_{\mathbf{q} i}^{\mathrm{Na} ; \mathrm{T}}=\left(\frac{\mathbf{q} \cdot \mathbf{e}_{\mathbf{q} i}^{\mathrm{Na}}}{|\mathbf{q}|} \frac{\mathbf{q}}{|\mathbf{q}|}, 0\right) \\
\mathbf{p}_{\mathbf{q} i}^{\mathrm{Na} ; \mathrm{L}}=\left(\mathbf{e}_{\mathbf{q} i}^{\mathrm{Na}}-\frac{\mathbf{q} \cdot \mathbf{e}_{\mathbf{q} i}^{\mathrm{Na}}}{|\mathbf{q}|} \frac{\mathbf{q}}{|\mathbf{q}|}, 0\right) .
\end{gathered}
$$

Unless otherwise stated, all simulations are based on the transverse, projected DOS, which yields excellent agreement with experiment. See Fig. 3 in the main text.

$$
k_{n, n+1}=\frac{\mu^{\prime 2}\langle n|x| n+1\rangle^{2}}{\hbar \cdot \arccos \left(e^{-1 / 2}\right)\left(4 \pi \varepsilon_{0}\right) R^{3}} .
$$


For two nearest neighbor $\mathrm{CO}$ molecules, the non-resonant rate constants $k_{n 1}$ are on the order of $10^{7}$ to $10^{8} \mathrm{~s}^{-1}$. The resonant rate constants are much larger, e.g. $k_{01}=1.8 \times 10^{11} \mathrm{~s}^{-1}$. The high resonant rate constants allow for fast diffusion of single vibrational states but significantly slow down the kMC simulations. To reduce the computation time, the resonant rate constants are scaled by a factor of $1 / 100$. No notable change in the vibrational energy distribution was found in test simulations with a scaling factor of $1 / 10$.

\section{The Skinner-Tully model of $\mathrm{CO}$ vibrational energy transfer to the $\mathrm{NaCl}$ substrate}

Fig. S6A depicts the ST model used for describing $\mathrm{CO}$ vibrational energy transfer to the $\mathrm{NaCl}$ substrate $(14,15,17,18)$.

In this model, we calculate the non-radiative $\mathrm{CO}-\mathrm{NaCl}$ energy transfer rate constants for a given vibrational state $n$ as (in SI units)

$$
\begin{gathered}
k_{\mathrm{nr}}^{\mathrm{ST}}=\sum_{p=1}^{p_{\max }} k_{n}^{(p)} \\
k_{n}^{(p)}=\frac{1}{\hbar}|\langle n|x| n-1\rangle|^{2}\left[n\left(\frac{E_{n}}{p}\right)+1\right]^{p} \frac{f_{p}^{2} I_{p}}{E_{n} p !} \\
f_{p}=(-1)^{p}\left(2^{p+1}-2\right) D^{\prime} \alpha^{\prime p+1} a_{o}^{p} \frac{m_{\mathrm{O}}}{M_{\mathrm{CO}}} \\
I_{p}=\lambda^{p} \frac{E_{n}}{h c \tilde{v}_{\mathrm{D}}} \sqrt{\frac{75 \pi}{p}} \exp \left[-\frac{75}{4 p} \cdot\left(\frac{E_{n}}{h c \tilde{v}_{\mathrm{D}}}-\frac{4 p}{5}\right)^{2}\right]
\end{gathered}
$$

where $E_{n}$ is the energy dissipated to phonons. The sum in Eq. 14 is truncated at $p_{\max }=$ $\operatorname{int}\left(E_{n} / \omega_{D}\right)+11$, since processes involving more phonons do not yield any further significant contributions. $m_{\mathrm{O}}$ and $m_{\mathrm{CO}}$ are the masses of ${ }^{18} \mathrm{O}$ and ${ }^{13} \mathrm{C}^{18} \mathrm{O}$, respectively, $D^{\prime}=10.917 \mathrm{eV}$ and $\alpha^{\prime}=2.34 \AA^{-1}$ are the parameters of the Morse-type CO-NaCl adsorption potential, $a_{0}$ is the Bohr radius, $\lambda=0.522$ an empirical parameter describing the global system-bath coupling, and $\tilde{v}_{\mathrm{D}}=$ $223 \mathrm{~cm}^{-1}$ the Debye frequency of $\mathrm{NaCl}$ in $\mathrm{cm}^{-1}$ (14). Other quantities are the same as in Section F.1 and F.2. Note that the original work by Corcelli and Tully contains two typographical errors in the formula for $I_{p}$. The corrected equations from Ref. (16) are used, where we would like to point out another small typographical error in the formula for $k_{n}^{(p)}$.

The ST model predicts a strong vibrational state dependence (v-dependence) of $k_{\mathrm{nr}}$ as shown in Fig. S4. The rate constants increase by more than five orders of magnitude between $v=1$ and $\mathrm{v}=25$. Note that the Skinner-Tully model relies on a coupling strength parameter, $\lambda$, which was deduced empirically by matching the total fluorescence decay time constant $(\tau=4.3 \mathrm{~ms})$ reported by Chang and Ewing (11) to kMC simulations in Ref. (14). According to Eq. 14-17, the absolute magnitude of $k_{\mathrm{nr}}^{\mathrm{ST}}$ is extremely sensitive to $\lambda$. However, we found that the dependence on $\lambda$ is quite similar for all the v states; hence, we expect that the strong v-dependence of $k_{\mathrm{nr}}^{\mathrm{ST}}$ shown in Fig. S4 remains at all values of $\lambda$. Note also that adjusting the Debye frequency in Eq. 17 has a similar effect. 


\section{The Chance-Prock-Silbey model of $\mathrm{CO}$ vibrational energy transfer to the $\mathrm{NaCl}$ substrate}

The CPS model (Fig. 6B) differs markedly from the ST model and has been described in detail in several seminal papers $(19,20,42,43)$. In particular, we recommend the review of Ref. (19). CPS is based on electromagnetic coupling to the solid, which absorbs and reflects light at the CO transition frequency, while the ST model relies on anharmonic coupling via the $\mathrm{CO}-\mathrm{NaCl}$ surface bond. CPS was originally developed to explain the experimentally observed changes in fluorescence lifetime of an electronically excited molecule as its distance from a metallic surface is varied $(24,26,29)$. Experimental control of the distance was accomplished by the introduction of inert organic spacer layers in the form of Langmuir-Blodgett-Kuhn films (44). Kuhn initially described the variation of lifetime with distance as a dipole emitter interacting with its electromagnetic echo field - i.e. the field reflected from the mirror. In his view, lifetime change is essentially a retardation effect where interference occurs between the emitting dipole and the image dipole set up in the solid. CPS showed that additional effects become important when the molecule comes close to the mirror, specifically creation of dipole-carrying excitations (e.g. plasmons and optical phonons) that are linked to the imaginary part of the solid's refractive index, $\kappa$. Working with a mathematical formalism written down to describe radio transmission in the presence of a partially conducting Earth (45), one that had its origins in Sommerfeld's ground wave paper (21), CPS were able to derive an exact expression within the classical limit that was valid at all distances (20) and gave excellent agreement with experiment (43). The most important extension in the theoretical treatment of CPS was to separate the lifetime of the dipole emitter into a radiative component, essentially the fluorescence lifetime, and a non-radiative component, where energy is transferred to the solid.

In the limit of small distances ( $d \rightarrow 0$ ), the CPS model provides an analytic solution for the nonradiative energy transfer rate to the solid. The rate constant, $k_{\mathrm{nr}}^{\mathrm{CPS}}$, is directly proportional to the square of the transition dipole moment of the molecule, which is proportional to $k_{\mathrm{f}} / \tilde{v}^{3}$, as well as the imaginary part of the index of refraction, $\kappa(19,46)$.

$$
\frac{k_{\mathrm{nr}}^{\mathrm{CPS}}}{k_{\mathrm{f}}}=\frac{3 \theta n \kappa}{16 \pi^{3} \tilde{v}^{3}|\epsilon+1|^{2} d^{3}} .
$$

where $\tilde{v}$ is the $\mathrm{CO}$ emission frequency in $\mathrm{cm}^{-1}, \epsilon=(n+\mathrm{i} \kappa)^{2}$ is the frequency dependent complex dielectric constant of $\mathrm{NaCl}$ at the emission frequency, $\theta$ is the orientation parameter and is 1 for perpendicular dipole orientation, and $d$ is the molecule's distance from the surface. For $\mathrm{CO}$ on $\mathrm{NaCl}: d=R_{\mathrm{C}-\mathrm{Na}}+M_{\mathrm{O}} /\left(M_{\mathrm{O}}+M_{\mathrm{C}}\right) R_{\mathrm{C}-\mathrm{O}}=3.36 \AA$ and is the distance of the CO center-ofmass to the $\mathrm{NaCl}$ interface, where $R_{\mathrm{C}-\mathrm{Na}}=2.7 \AA$ and $R_{\mathrm{C}-\mathrm{O}}=1.14 \AA$ (47). $M_{\mathrm{O}}$ and $M_{\mathrm{C}}$ are the masses of ${ }^{18} \mathrm{O}$ and ${ }^{13} \mathrm{C}$, respectively. The refractive index $n$ and the extinction coefficient, $\kappa$, are almost constant $\left(n=1.52\right.$ and $\left.\kappa=1.8 \times 10^{-9}\right)$ in the mid-IR wavelength range relevant to our work $\left(1500-2600 \mathrm{~cm}^{-1}\right)(48)$. While $\kappa$ is quite small $-\mathrm{NaCl}$ is nominally transparent in the mid-infrared, this value is still large enough to have a clearly observable effect. We then calculate the vdependent rate constants using the calculated $\mathrm{CO}$ gas phase fluorescence rates $k_{\mathrm{f}}(\mathrm{v})$ shown in Fig. $\mathrm{S} 4$ and the fundamental emission frequencies $\tilde{v}(\Delta \mathrm{v}=1)$ of the monolayer ${ }^{13} \mathrm{C}^{18} \mathrm{O}$ determined from Eq. 1.

The calculated values for $k_{\mathrm{nr}}^{\mathrm{CPS}}$ in Fig. S4 show a much weaker v-dependence than $k_{\mathrm{nr}}^{\mathrm{ST}}$, which is closely related to the $\mathrm{v}$-dependence of the radiative rate constants $k_{\mathrm{f}}$. This is a qualitative characteristic of the CPS model reflecting coupling via the electromagnetic field. This aspect of the CPS improves our confidence that even a more sophisticated treatment of the ST model or 
534 another model based on mechanical coupling via the anharmonic potential energy surface would 535 fail to agree with our experimental observations (Fig. 4B Main text). The ratio $k_{\mathrm{nr}}^{\mathrm{CPS}} / k_{\mathrm{f}}$ changes 536 from $5(\mathrm{v}=1)$ to $13(\mathrm{v}=27)$ due to the $\tilde{v}^{-3}$ dependence of Einstein A-coefficients, $k_{\mathrm{f}}$, appearing in 537 Eq. 18. The CPS model also predicts a reduction of the fluorescence rate by $10 \%$ due to interference 538 of the emitted photon with itself upon reflection from the surface. Since $k_{\mathrm{f}}$ is about one order of 539 magnitude smaller than $k_{\mathrm{nr}}^{\mathrm{CPS}}$, this effect on the emission rate is neglected in our simulations.

\section{Vibrational-to-electronic (V-E) energy transfer rate constants} $\mathrm{CO}\left(\mathrm{X}^{1} \Sigma^{+}, \mathrm{v}=28\right)$ is $6.01 \mathrm{eV}$, in close resonance with the vibrational ground state of the first electronic excited state $(6.01 \mathrm{eV})$ of $\mathrm{CO}$ molecules in the gas phase. Thus, we assume a V-E energy loss channel in the kMC simulation. We set the corresponding rate constants to $1 \times 10^{5} \mathrm{~s}^{-1}$ for vibrationally excited states above $v=27$, which avoids accumulation of population in these states. 


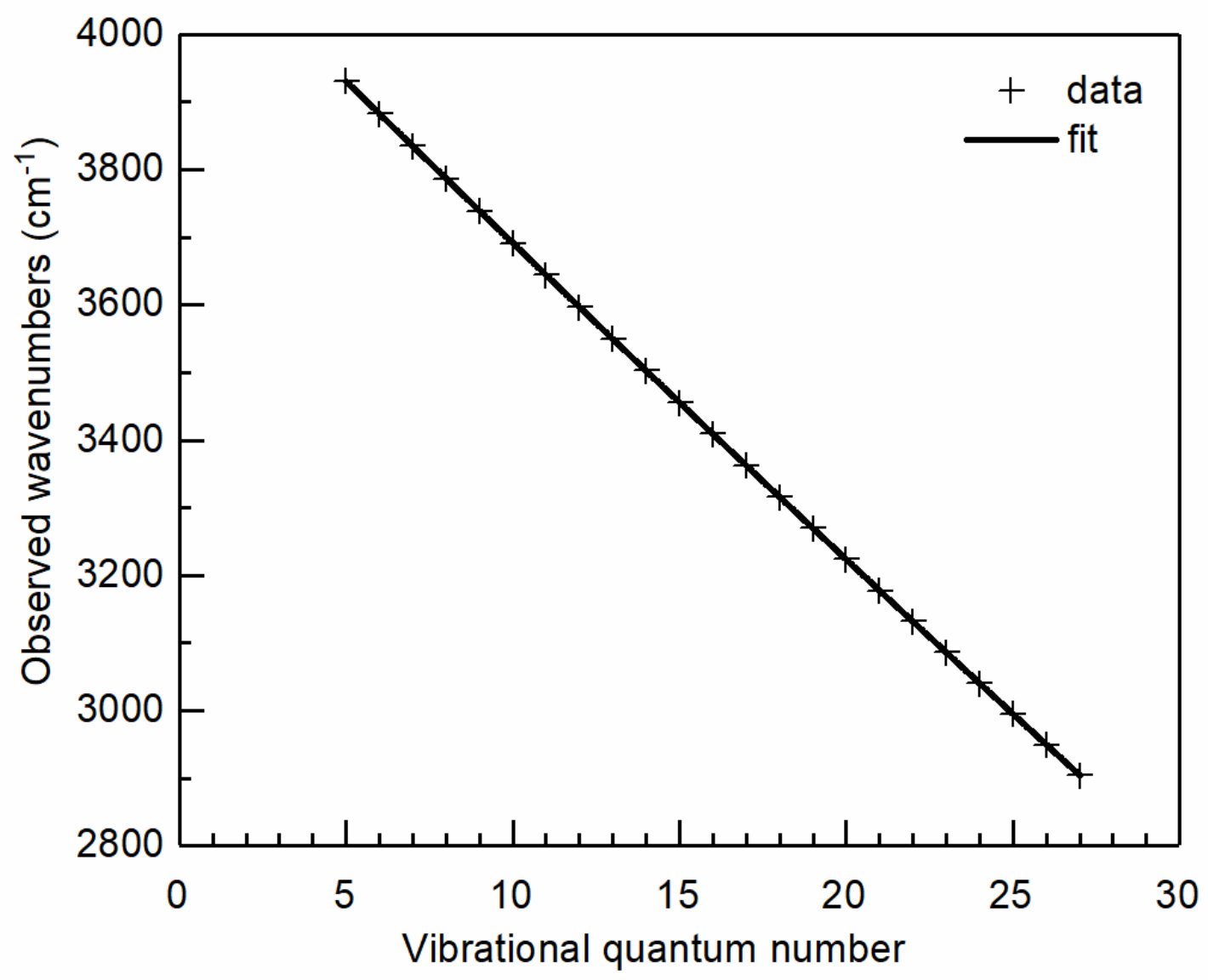
(100), see Fig. 2 in the main manuscript. The solid line is a least-square fit to the data based on Eq. 2. 
A

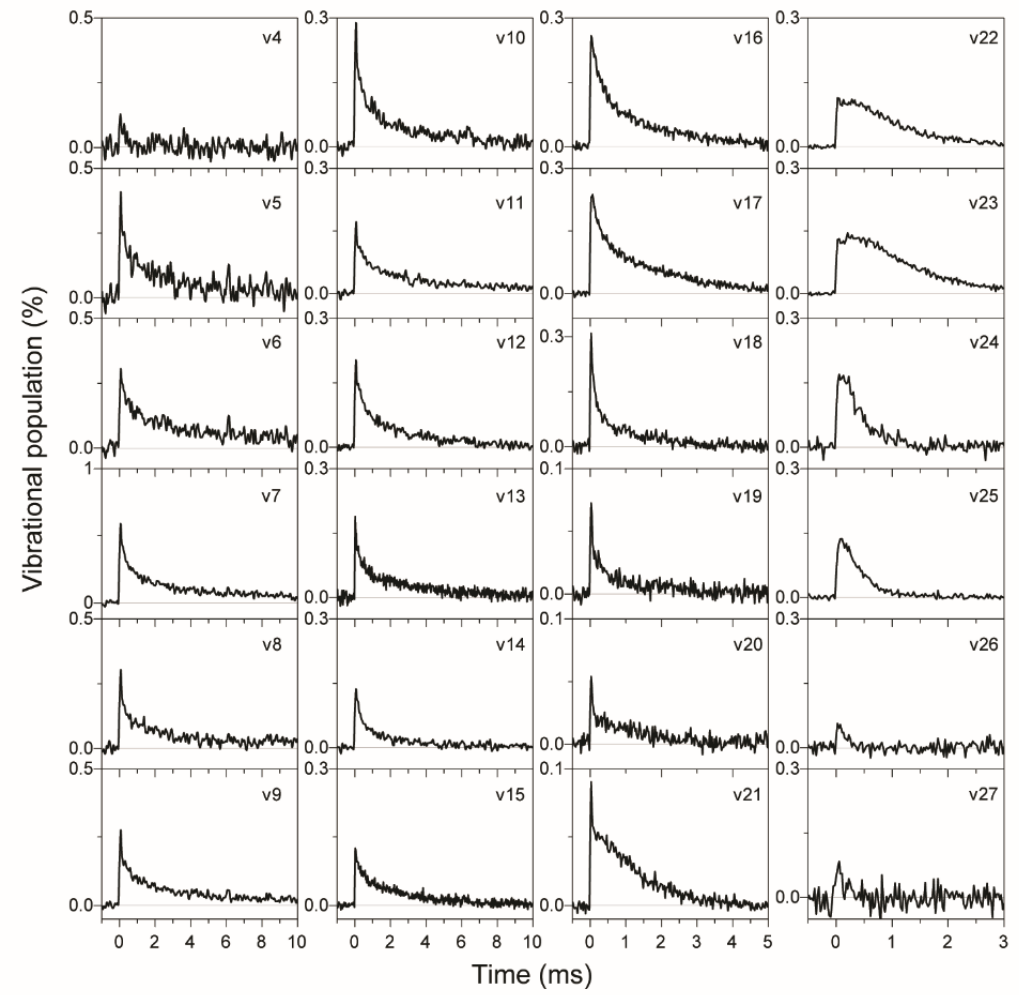

B

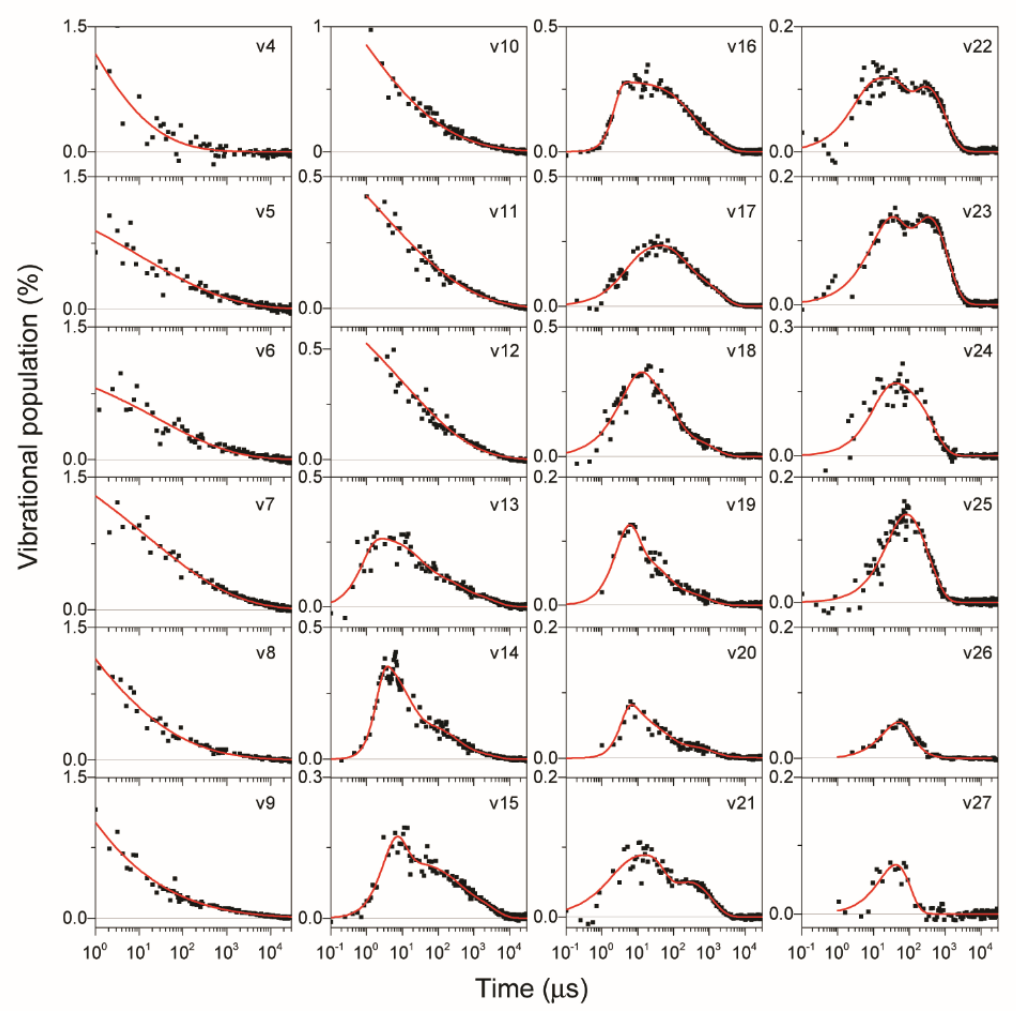

Figure S2 | Temporal profiles of the CO monolayer overtone emission for the vibrational states 4-27 at $7 \mathrm{~K}$. Panel A and B show the temporal profiles on a linear and logarithmic time-scale, respectively. Note the different time ranges for different vibrational states. The vibrational population shown on the $y$-axis is calibrated according to Section E. Panel A: Temporal profiles are shown with a bin-time of $25 \mu$ s. Panel B: 


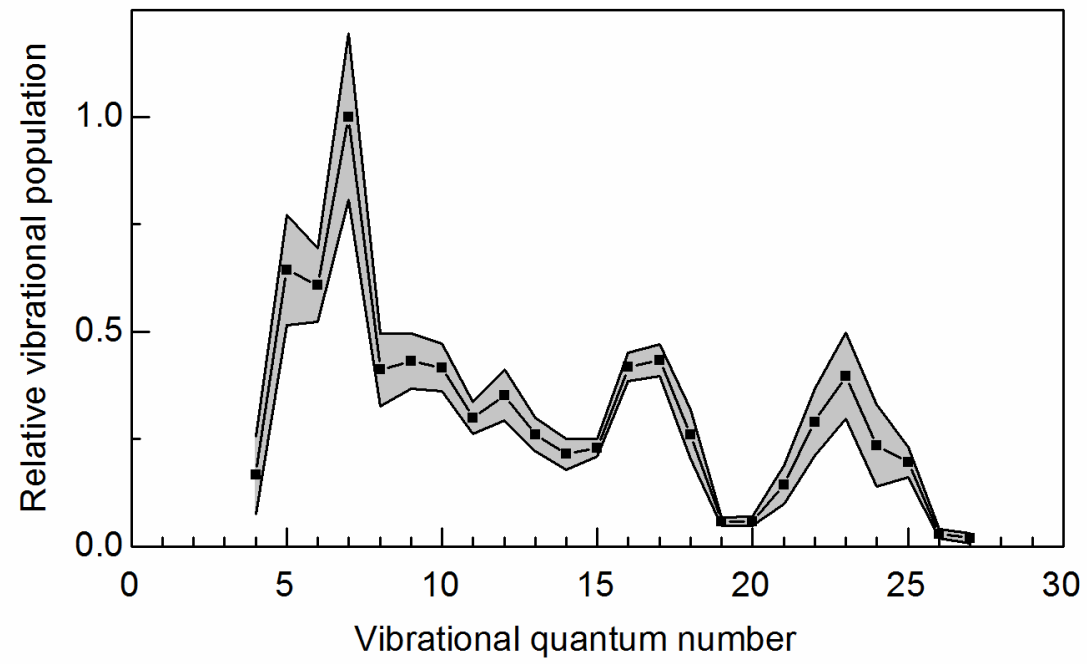

Figure S3 | The relative vibrational population distribution of monolayer CO integrated over 0.05-

$560 \quad \mathbf{1 . 0 5}$ ms (black dots). The grey shaded region represents the overall uncertainty. 


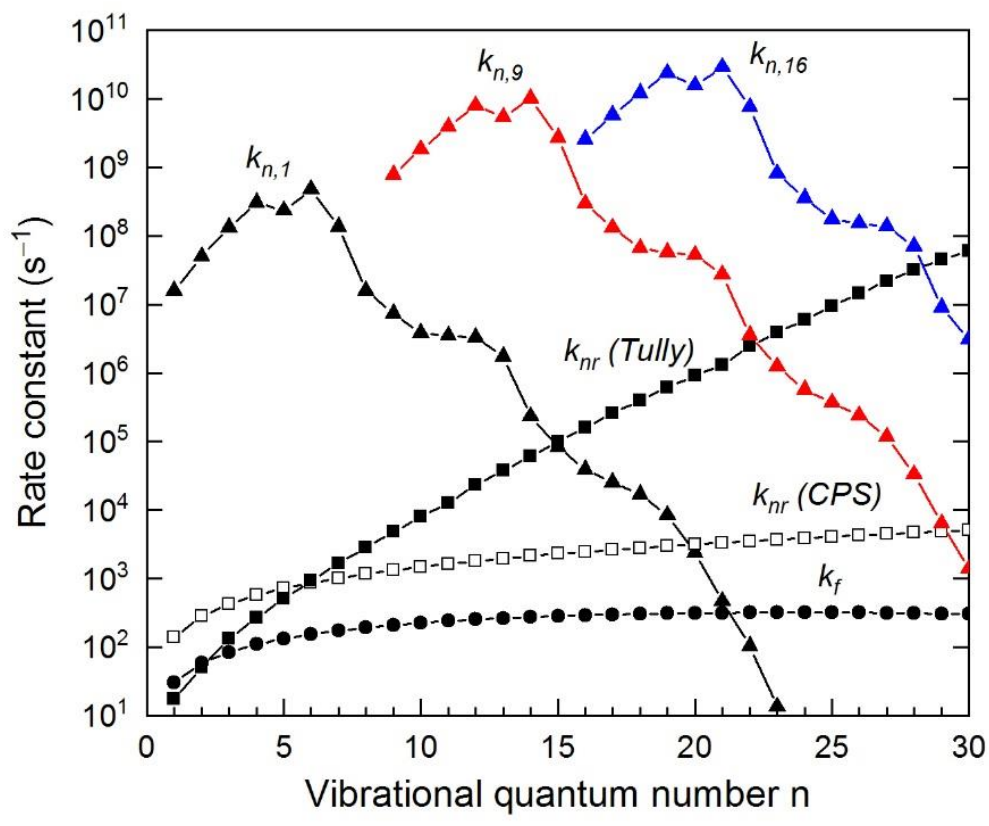

Figure S4 | Rate constants calculated for the kMC simulations. Shown are rate constants for singlequanta processes: fluorescence (filled circles), nearest neighbor vibrational energy pooling $\mathrm{CO}(\mathrm{n})+\mathrm{CO}(\mathrm{m})$ $\rightarrow \mathrm{CO}(\mathrm{n}+1)+\mathrm{CO}(\mathrm{m}-1)$ for selected states $\mathrm{m}=1,9$ and 16 (triangles), and non-radiative relaxation for the Tully (filled squares) and the CPS model (open squares). Note that the VEP rate constants depend strongly on distance (see Section F.2 for details). 


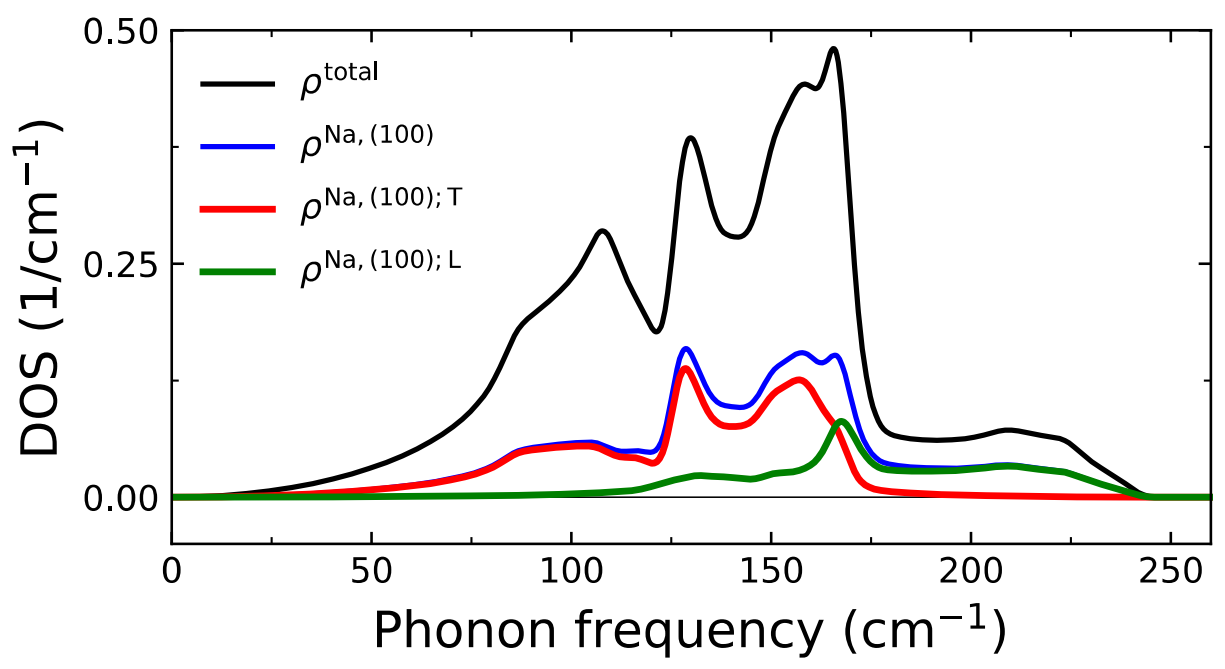

Figure S5 | Phonon density of states (DOS) for bulk NaCl. Shown are the total DOS (black solid line), the DOS projected onto phonons which include Na-atom displacements in the (100) plane (blue) together with its decomposition into transverse ( $\mathrm{T}$, red) and longitudinal (L, green) phonons as defined by Eq. 10-12 in the text. The DOSs are plotted as a function of phonon frequencies corresponding to the phonon excitation energies $E$. The total DOS is normalized to 1, and the transverse and longitudinal projections sum up to to the Na-atom-(100) projection. 
A

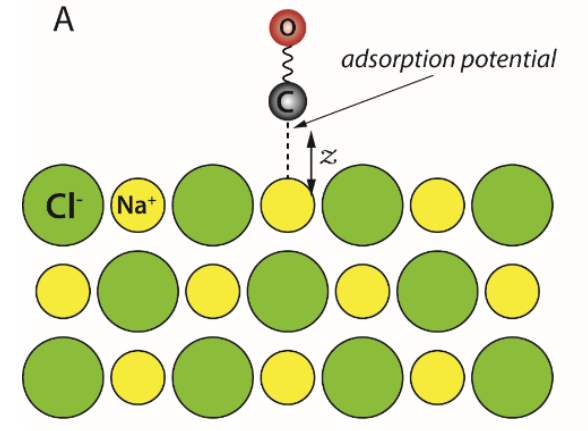

B

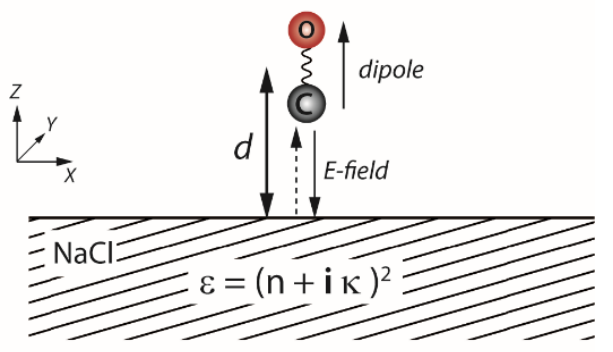

Figure S6 | Theoretical models for describing vibrational energy transfer of adsorbed CO molecules to the solid NaCl substrate. (A) Tully model: Vibrational energy flows by mechanical coupling of the $\mathrm{CO}$ oscillator to the bulk $\mathrm{NaCl}$ phonon bath by anharmonic interaction through the $\mathrm{C}-\mathrm{Na}^{+}$bond. (B) $\mathrm{CPS}$ model: Vibrational energy flows from the molecule to the solid via the electromagnetic field emitted by the oscillating $\mathrm{CO}$ dipole near the $\mathrm{NaCl}$ half-space with a complex frequency dependent dielectric constant. Note that the CPS model is not defined in terms of atoms and forces between them. 